\title{
Hepatocarcinoma como causa de abdome agudo em adolescente: relato de caso
}

\author{
Acute abdomen caused by rupture of hepatocellular carcinoma in an adolescent: \\ case report
}

João Augusto dos Santos Martines ${ }^{a}$, Brenda Margatho Ramos Martinesa, Daniel Gonçalves Leal Araújob $^{b}$ Fabiana Roberto Limac

Martines JAS, Martines BMR, Araújo DGL, Lima FR. Hepatocarcinoma como causa de abdome agudo em adolescente: relato de caso. Autopsy Case Rep [Internet]. 2011;1(2):49-56.

\section{RESUMO}

\begin{abstract}
O carcinoma hepatocelular $(\mathrm{CHC})$ é pouco prevalente nos países ocidentais, porém é um dos tumores mais freqüentes na Ásia e a quinta causa de câncer no mundo. É relatado um caso de adolescente do sexo feminino sem fatores de risco para um tumor extremamente agressivo, internada no Hospital Universitário da Universidade de São Paulo. A apresentação clínica foi de abdome agudo secundário a sangramento para cavidade peritoneal por ruptura do tumor evidenciado através de tomografia computorizada multidetectores (TCMD) confirmado por laparotomia exploradora e biópsia hepática e tratada com rafia hemostática do parênquima hepático.
\end{abstract}

Unitermos: Carcinoma hepatocelular; Abdome agudo; Hemorragia.

\begin{abstract}
Hepatocelular carcinoma (HCC ) is not frequent in West Countries, but is one of the most prevalent tumors in Asia and the fifth most common cancer in the world. We present a case of a teenager girl diagnosed with an extremely aggressive HCC without the known risk factors. The patient was admitted in Hospital Universitário da Universidade de São Paulo with the diagnosis of acute hemorrhagic abdomen secondary to hepatic rupture confirmed by exploratory laparotomy and liver biopsy, treated with surgical hemostasis of the ruptured hepatic parenchyma.
\end{abstract}

Keywords: Hepatocellular carcinoma; Acute haemorrhagic abdomen; Haemorrhagia.

\footnotetext{
a Serviço de Iconologia do Hospital Universitário - Universidade de São Paulo, São Paulo/SP, Brasil.

b Instituto do Coração do Hospital das Clínicas da Faculdade de Medicina - Universidade de São Paulo, São Paulo/SP, Brasil.

c Serviço de Anatomia Patológica do Hospital Universitário - Universidade de São Paulo, São Paulo/SP, Brasil.
} 


\section{Relato do Caso}

Paciente de 16 anos de idade, sexo feminino, foi admitida no Pronto-Socorro do Hospital Universitário da Universidade de São Paulo (HU-USP) com queixa de dor abdominal há 01 mês e constipação com piora progressiva, mas negava emagrecimento. Ao exame físico a paciente encontrava-se eutrófica, sonolenta, descorada, anictérica, $P A=104 \times 50$ $\mathrm{mmHg}$, Pulso $=120$ bpm, Freqüência respiratória $=24$ mrpm. Apresentava abdome distendido, com intensa dor difusa a palpação e fígado palpável a $10 \mathrm{~cm}$ abaixo do rebordo costal direito. O exame do coração e pul- mões não apresentava alterações propedêuticas. Foi aventada a hipótese de abdome agudo inflamatório e solicitada ultra-sonografia, que evidenciou acentuada hepatomegalia à custa de múltiplas massas e nódulos hepáticos, além de moderado volume de líquido livre na cavidade abdominal, com aspecto espesso na pelve, sugerindo hemoperitônio.

Os exames laboratoriais de entrada estão apresentados na Tabela 1.

Tabela 1 - Exames laboratoriais

\begin{tabular}{llllll}
\hline Hemoglobina & $\mathrm{g} / \mathrm{dl}$ & 7,8 & Glicose & $\mathrm{mg} / \mathrm{dl}$ & 137 \\
Hematócrito & $\%$ & 23 & Bilirrubinas totais & $\mathrm{mg} / \mathrm{dl}$ & 1,4 \\
Leucócitos & $/ \mathrm{mm} 3$ & 13800 & Bilirrubina direta & $\mathrm{mg} / \mathrm{dl}$ & 0,8 \\
Bastonetes & $\%$ & 1 & $\mathrm{ALT}$ & $\mathrm{U} / \mathrm{l}$ & 147 \\
Segmantados & $\%$ & 66 & $\mathrm{AST}$ & $\mathrm{U} / \mathrm{l}$ & 373 \\
Eosinófilos & $\%$ & 2 & $\mathrm{INR}$ & & 1,0 \\
Basófilos & $\%$ & 1 & Uréia & $\mathrm{mg} / \mathrm{dl}$ & 44 \\
Linfócitos & $\%$ & 24 & Creatinina & $\mathrm{mg} / \mathrm{dl}$ & 0,7 \\
Monócitos & $\%$ & 6 & & & \\
Plaquetas & $/ \mathrm{mm} 3$ & 880000 & & & \\
\hline
\end{tabular}

A endoscopia digestiva alta mostrou compressão extrínseca do fundo gástrico. As Sorologias para vírus da hepatite $\mathrm{B}$ e $\mathrm{C}$ foram negativas e a paciente apresentava IgG para vírus da hepatite $A$.

A dosagem da alfa feto proteína resultou em 60500,0 ng/ml (valor normal até $10 \mathrm{ng} / \mathrm{ml}$ ).

A TCMD do fígado apresentava dimensões acentuadamente aumentadas, contornos lobulados e com atenuação difusamente heterogênea à custa de múltiplos nódulos e massas disseminadas pelo parênquima (Figura 1). Algumas massas apresentavam realce precoce arterial, outras com conteúdo hiperatenuante de aspecto hemorrágico. O estudo dinâmico com contraste endovenoso mostrava realce vascular anômalo com extravasamento do mesmo, formando nível líquido-líquido, indicando sangramento ativo na cavidade abdominal (Figuras 2 e 3). Também havia sinais de hemoperitônio de grande volume, formando "efeito de hematócrito" na pelve (Figura 4). 


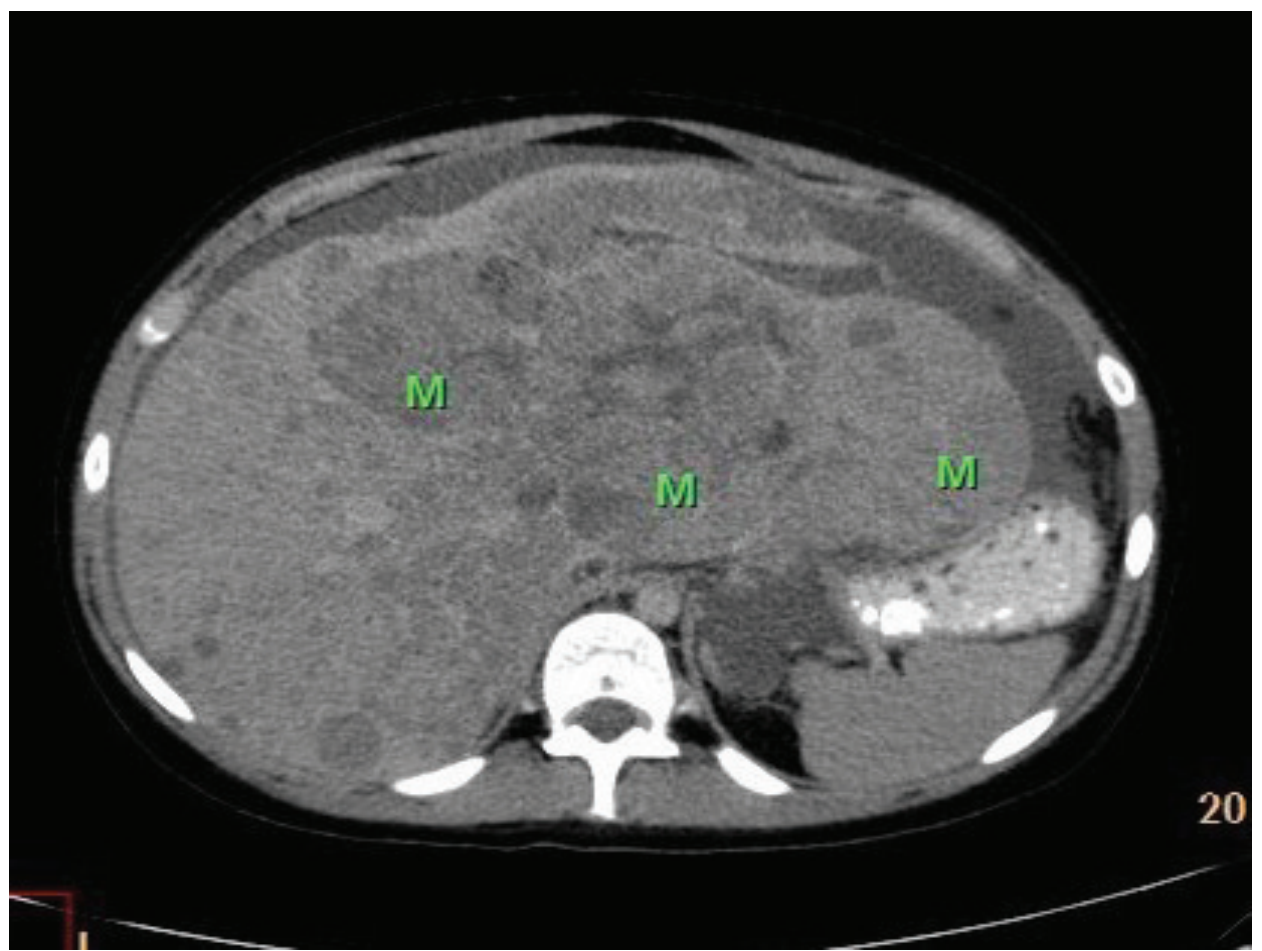

Figura 1 - Corte Axial - Massas hepáticas heterogêneas (M), confluentes, com realce heterogêneo ao meio de contraste. Foi aventada hipótese de metástases extra-hepáticas.

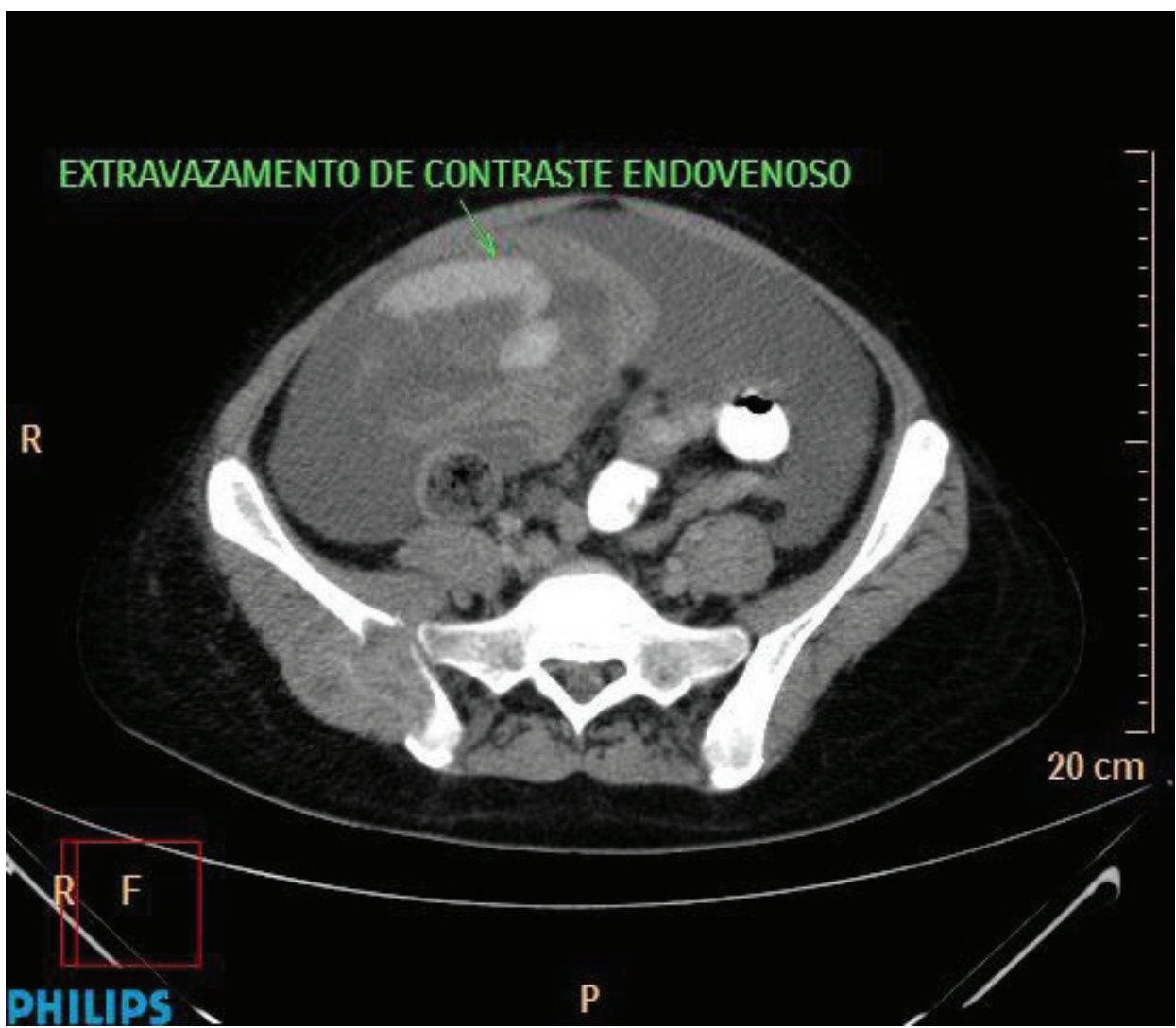

Figura 2 - Corte Axial - Extravasamento de contraste endovenoso para cavidade peritoneal, evidenciando ruptura hepática, uma das possíveis complicações do CHC avançado. 


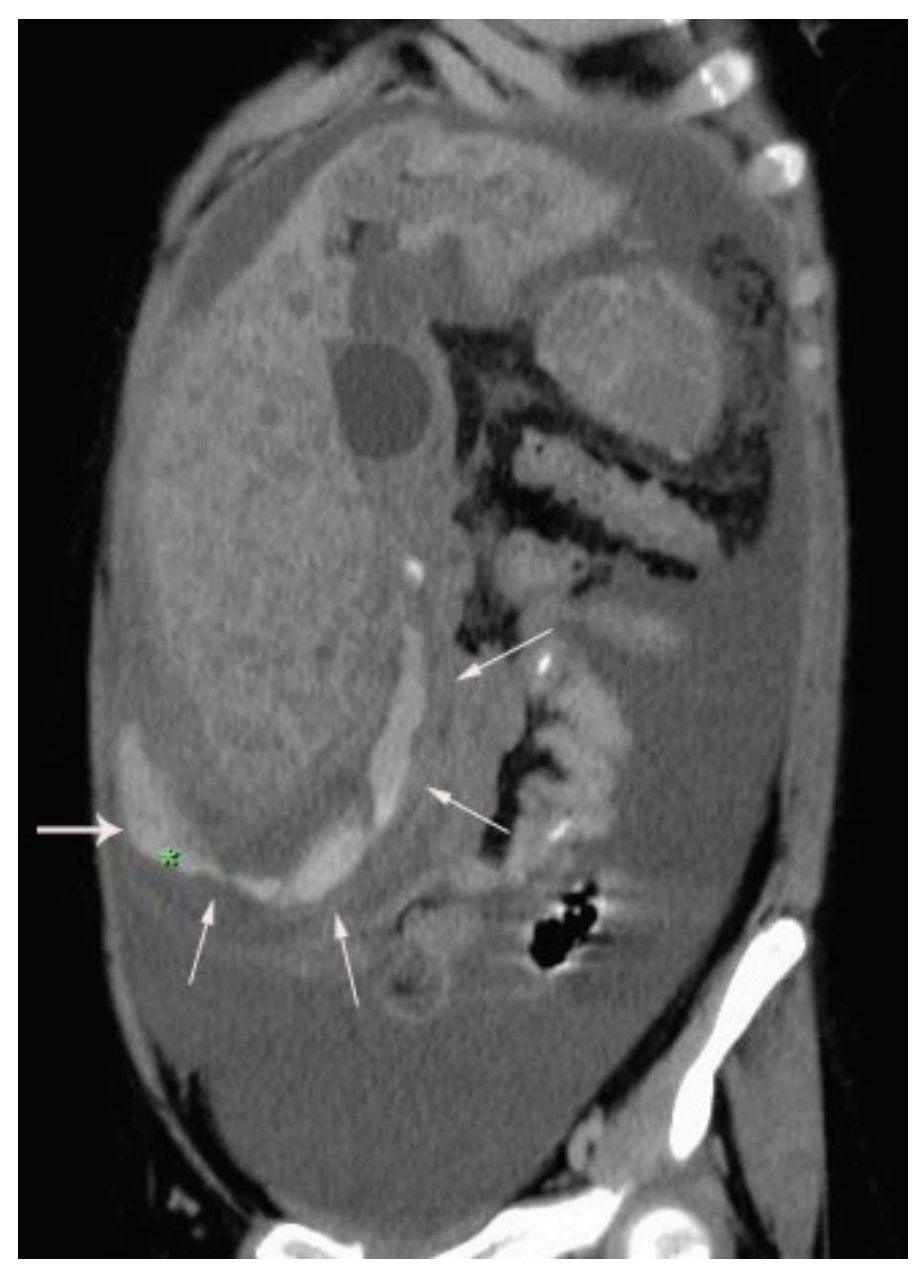

Figura 3 - Reformatação coronal - Extravasamento de contraste endovenoso (setas) para cavidade abdominal, compatível com ruptura hepática.

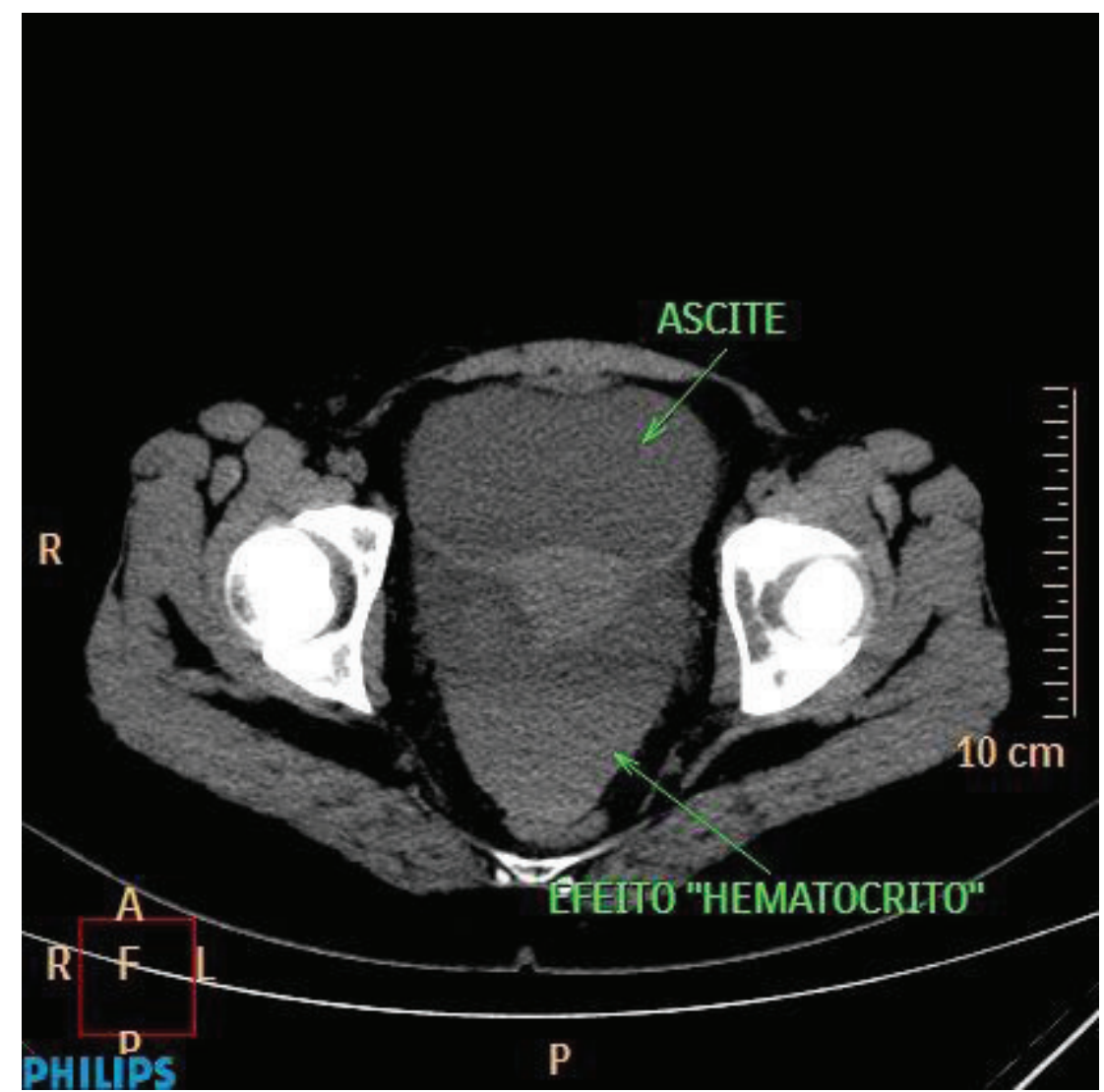

Figura 4 - Corte axial - Líquido mais denso em fundo de saco, caracterizando o efeito hematócrito. 
O estudo com janela óssea mostrava lesões osteolíticas compatíveis com metástases na bacia (Figura 5).

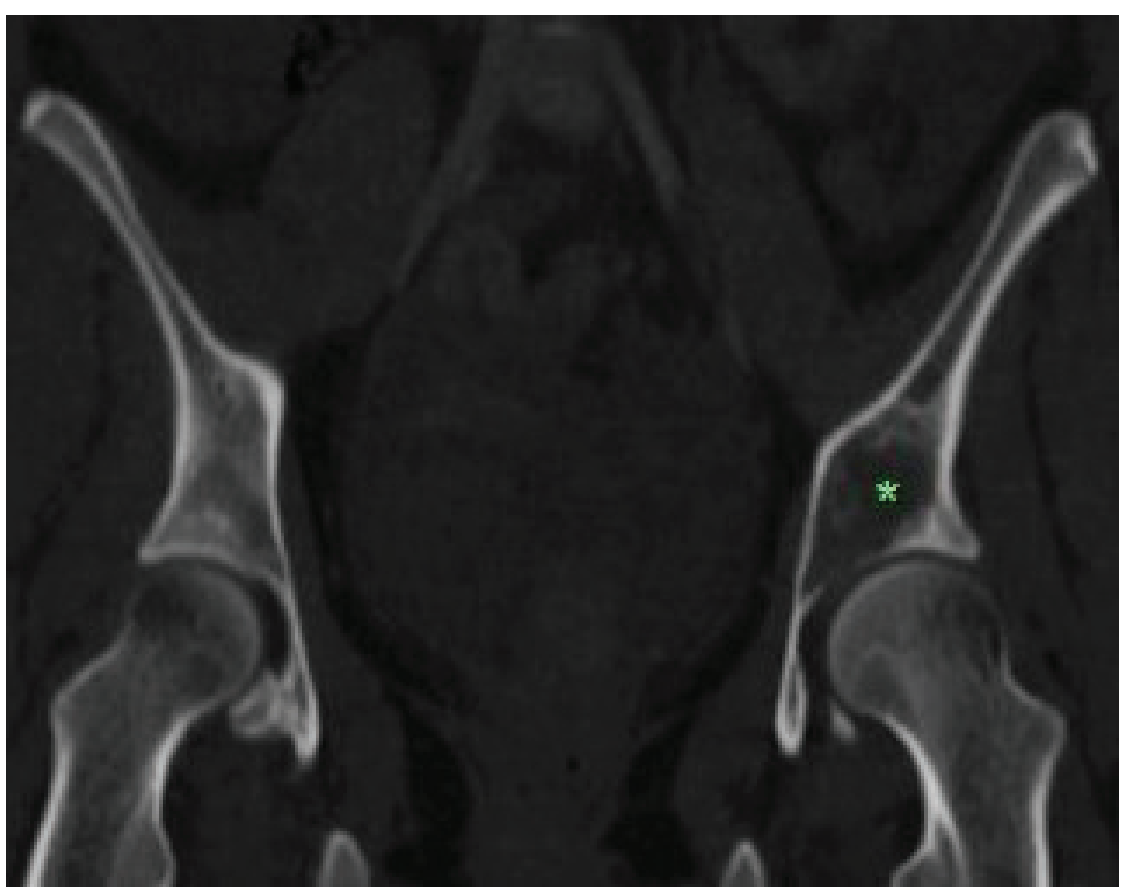

Figura 5 - Reformatação coronal - Lesão osteolítica no quadril esquerdo. Metástases ósseas são comuns no CHC avançado.

A paciente foi submetida à laparotomia exploradora que confirmou os achados tomográficos, identificando área de laceração hepática, inúmeras lesões nodulares amareladas hipervascularizadas, além de lesão da parede gástrica de aproximadamente $15 \mathrm{~cm}$ de diâmetro, sendo realizada hemostasia da laceração e biópsia hepática. Realizada endoscopia digestiva alta no intra-ope- ratório que não revelou lesão da mucosa gástrica. O resultado da histologia, seguida da avaliação imunohistoquímica, foi de carcinoma hepatocelular moderadamente diferenciado, grau III de Edmondson-Steiner, de padrão predominantemente trabecular, com focos necróticos, em fígado não cirrótico (Figuras 6 e 7 ).

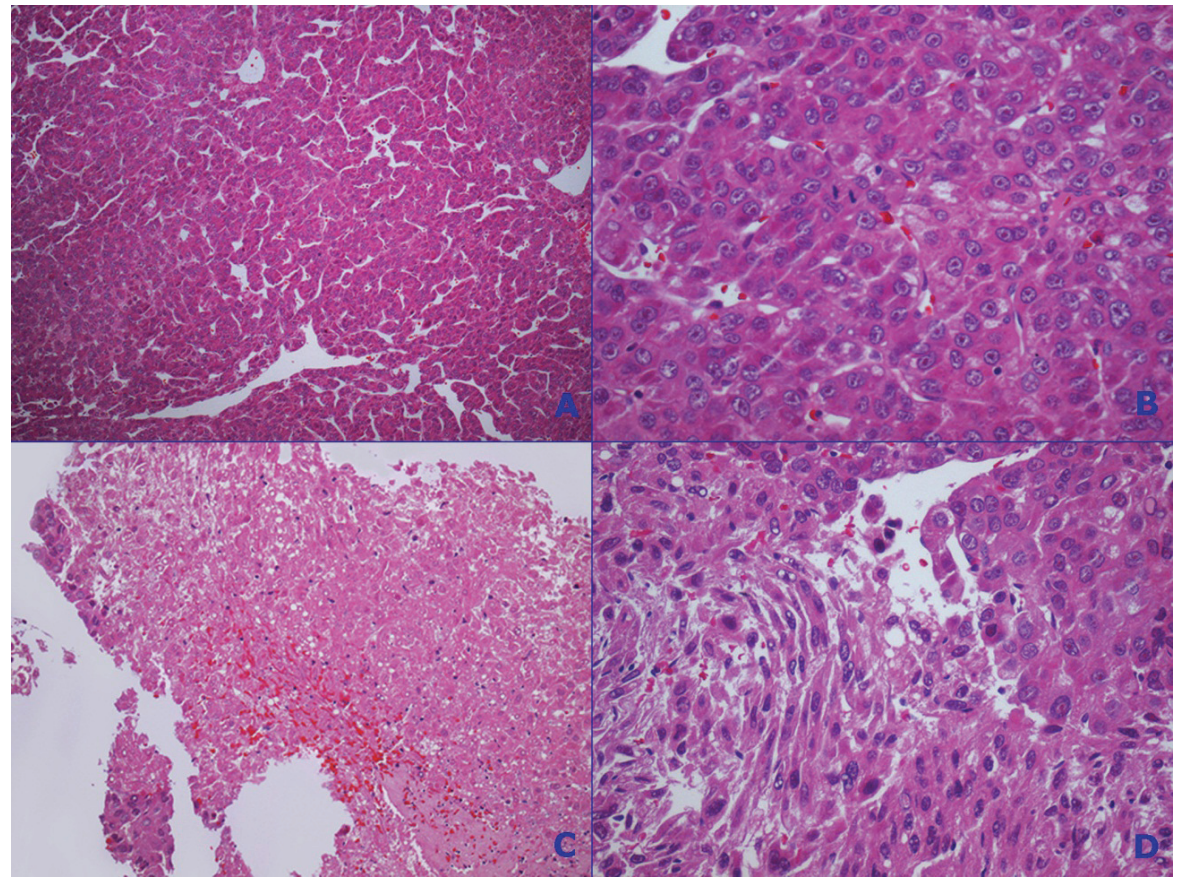

Figura 6 - Fotomicrografias - A - (HE 100X) Carcinoma hepatocelular (CHC) moderadamente diferenciado, com arranjo em trabéculas de espessura variável, separadas por espaços sanguíneos similares a sinusóides, B - (HE 400X) Células neoplásicas poligonais, com citoplasma eosinofílico volumoso, núcleos grandes vesiculados e nucléolos proeminentes. Presença de frequentes figuras de mitoses, C - (HE $100 \mathrm{X})$ - Área de necrose tumoral, acompanhada de algumas células tumorais à esquerda da foto, D - (HE - $400 \mathrm{X}$ )- Nota-se pequena área pouco diferenciada do carcinoma hepatocelular, caracterizada por células neoplásicas alongadas, com núcleos bem irregulares e ocasionais binucleação nuclear. Este aspecto focal assemelha-se morfologicamente a um carcinoma sarcomatóide. À direita da foto, há o padrão trabecular predominante deste $\mathrm{CHC}$. 


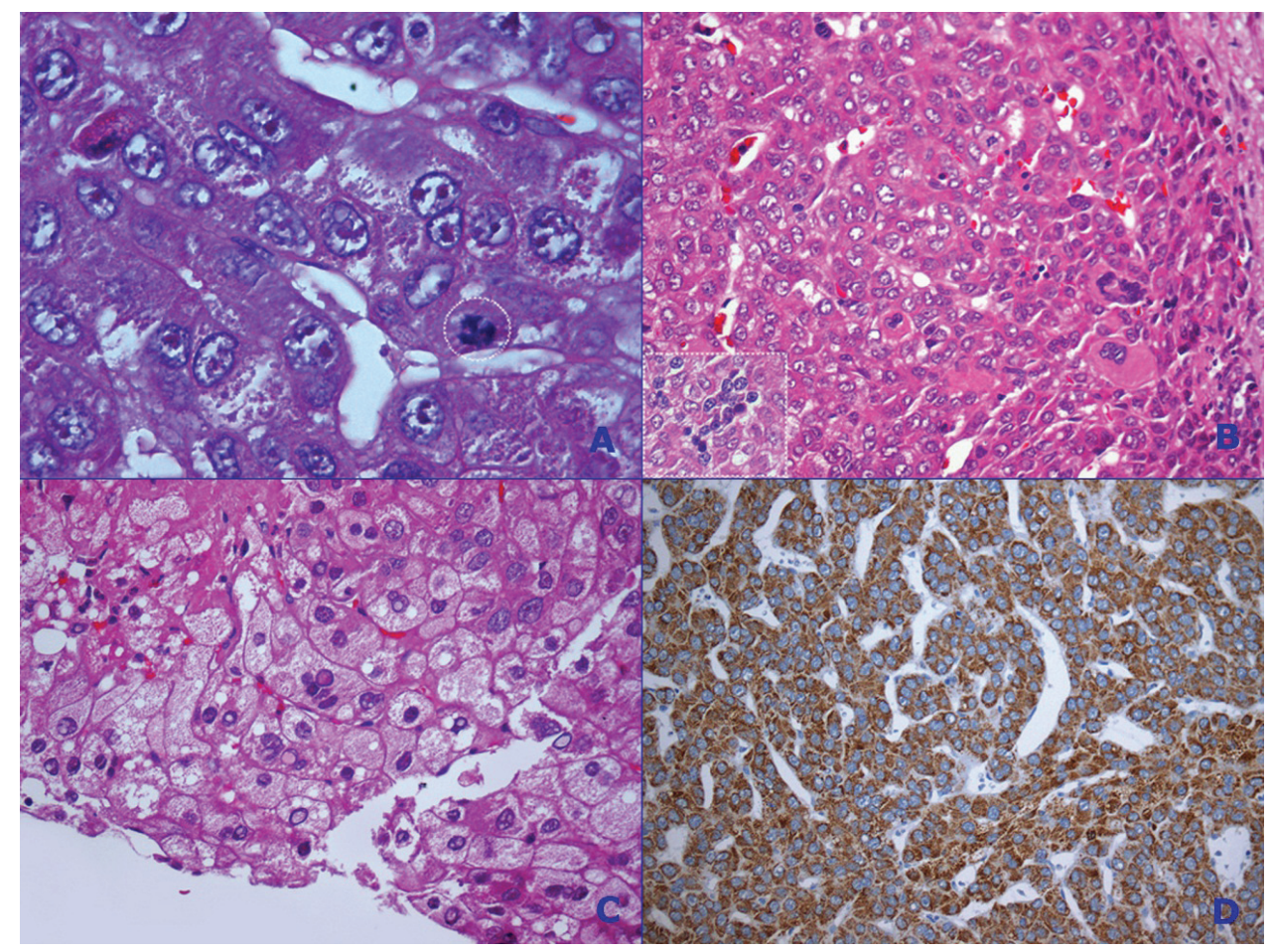

Figura 7 - Fotomicrografias - A (HE - imersão - 1000X) Imagem em grande aumento mostra uma mitose atípica tripolar em uma célula maligna (destacada dentro de círculo), além de detalhes morfológicos nucleares das células tumorais, como os nucléolos eosinofílicos e bem proeminentes, B - (HE $400 \mathrm{X}$ )- Em região focal do CHC, visualizamse raros focos pequenos de hematopoiese, como esse agregado sinusoidal de células hematopoiéticas no destaque do canto inferior esquerdo da foto. À direita, há 2 células grandes multinucleadas que são megacariócitos. A hematopoiese pode ocorrer no CHC, especialmente em indivíduos mais jovens, C - (HE - 400 X) - Algumas pseudoinclusões eosinofílicas nucleares e várias gotículas de esteatose, em células neoplásicas volumosas de citoplasma rarefeito, nessa área pequena de padrão sólido do carcinoma hepatocelular, D - (Imunohistoquímica - 200 X) - Positividade difusa e intensa do marcador imuno-histoquímico Hepatocyte confirmando a origem hepatocitária da neoplasia.

A paciente recuperou-se da laparotomia e da hemostasia hepática sendo encaminhada para oncologia clínica para prosseguir o tratamento. Sob cuidados paliativos veio a falecer após 3 meses do diagnóstico.

\section{DISCUSSÃO}

O carcinoma hepatocelular é a quinta mais frequente neoplasia no mundo responsável por 500.000 mortes anualmente, pouco prevalente nos países ocidentais, ocorrendo mais frequentemente $(>80 \%)$ na Africa sub-saariana ou em países da Ásia oriental. A China isoladamente apresenta mais de $50 \%$ de todos os casos do globo. ${ }^{1}$ A distribuição entre sexo mostra predominância para o sexo masculino variando de 2:1 a 4:1 dependendo da população de risco. Em regiões de baixo risco (Estados Unidos, Canadá e Reino Unido) o pico de incidência ocorre aos 75 anos ou mais e nas regiões de alto risco (população asiática) a faixa etária de ocor- rência para homens varia de 60 a 65 anos e para mulheres de 65 a 70 anos de idade. ${ }^{2}$ A ocorrência na população jovem é rara. Stroffolini et al estudou 1148 pacientes italianos com diagnóstico de $\mathrm{CHC} \mathrm{e}$ encontrou somente 1,3\% com idade entre 20 e 40 anos. ${ }^{3} \mathrm{Em}$ Taiwan foram registrados 122 casos histologicamente comprovados de $\mathrm{CHC}$ em crianças e adolescentes (faixa etária de 0 a 15 anos) num período de 13 anos. Nesta faixa etária a predominância apresentou 1 pico no primeiro ano de idade e outro aos 15 anos. Neste mesmo estudo Lee et al observou 43 casos de Hepatoblastoma que predominaram a faixa etária de 1 ano de idade. ${ }^{4} \mathrm{~A}$ associação da infecção pelo vírus $\mathrm{B}$ e o $\mathrm{CHC}$ nesta faixa etária é mais frequentemente observada até a idade de 4 anos, acredita-se que a infecção seja vertical na maior parte dos casos. ${ }^{4}$

Dentre a população adulta, 80 a $90 \%$ dos casos estão relacionados à infecção pelos vírus da hepatite $\mathrm{B}$ e $\mathrm{C}$ e à cirrose hepática secundária 
ao alcoolismo. Mais raramente, doenças de depósito, deficiência de alfa-1 antitripsina, ingestão de aflatoxinas, tirosinemia hereditária, hemocromatose hereditária e Doença de Wilson podem causar a doença. ${ }^{5}$

Embora a sintomatologia seja inespecífica e, muitas vezes, relacionada às doenças subjacentes do paciente, é descrita uma tríade clássica de dor abdominal no quadrante superior direito, emagrecimento e aumento do volume abdominal. Pode haver síndromes paraneoplásicas como febre, leucocitose, caquexia, eritrocitose, hipercalcemia, síndrome carcinóide, hipoglicemia e porfiria cutânea.

A dosagem da alfa-feto proteína nem sempre está elevada, porém é mais frequentemente alterada nos tumores grandes.

A evolução natural do $\mathrm{CHC}$ é o aumento progressivo da massa primária até que haja comprometimento da função hepática ou metástase, geralmente para os pulmões. As principais causas de morte decorrem de: a) caquexia; b) complicações de hipertensão portal como sangramento gastrointestinal ou por varizes esofágicas; c) insuficiência hepática ou encefalopatia hepática; d) ruptura do tumor com hemorragia fatal. ${ }^{6}$

Apesar do carcinoma hepatocelular ser um tumor de crescimento lento é geralmente fatal por não apresentar sintomas até que atinja estágios avançados da doença. ${ }^{1}$ Os sintomas mais freqüentes são: mal estar, perda de peso, dor abdominal, distensão abdominal, hepatomegalia, ascite, febre e icterícia. Hemoperitônio secundário a ruptura do hepatocarcinoma um sinal de doença avançada e pior prognóstico. ${ }^{7} \mathrm{Na}$ Ásia a incidência desta complicação varia de $14,5 \%$ em Hong Kong a $10 \%$ no Japão. Nos países com menor incidência de $\mathrm{CHC}$ esta complicação chega a 3\% como relatado no Reino Unido. ${ }^{8}$ Várias hipóteses foram aventadas para a ruptura espontânea, tais como localização subcapsular, grandes dimensões, associação com hipertensão portal, necrose tumoral, lesões vasculares causadas pelo tumor e obstrução venosa. ${ }^{9}$

No caso relatado não havia os reconhecidos fatores de risco para $\mathrm{CHC}$ e o hemoperitônio foi atribuído à laceração hepática devido às grandes massas tumorais. O prognóstico dos pacientes jovens com diagnóstico de $\mathrm{CHC}$ parece não diferir, porém há estudos que mostram a tendência de pior prognóstico devido ao estádio avançado da doença por ocasião do diagnóstico comparados com os pacientes adultos. ${ }^{10}$

A conduta terapêutica indicada para estes casos é a ressecção do tumor, quando possível. ${ }^{9}$ Nas últimas 2 décadas a mortalidade pós hepatectomia reduziu de 10\%-20\% observada nos anos 1980 para menos de $5 \%$ nos dias atuais. ${ }^{1}$ O tratamento curativo pode ser a ressecção segmentar, desde que seja possível preservar a atividade hepática normal adotando-se margens livres de $1 \mathrm{~cm}$, ausência de metástases, tamanho do fígado residual. ${ }^{1} \mathrm{Em}$ tumores maiores, o transplante ortotópico é a única opção de tratamento curativo. A sobrevida média sem tratamento é de apenas 6 a 20 meses.

Metástases ósseas, presente neste caso, representam segundo alguns estudos, $28 \%$ de todas as lesões à distância do $\mathrm{CHC}$, sendo o terceiro local mais comum, depois de pulmões e linfonodos abdominais. ${ }^{11}$

O caso relatado apresenta vários aspectos atípicos relacionados ao diagnóstico de $\mathrm{CHC}$, portanto ressaltamos a importância do método de imagem neste caso como recurso diagnóstico.

\section{AGRADECIMENTO}

Os autores agradecem a Rosa Maria da Conceição Zanardi pelo auxílio na documentação das imagens apresentadas.

\section{REFERÊNCIAS}

1. Song TJ, Ip EWK, Fong Y. Hepatocellular carcinoma: current surgical management. Gastroenterology. 2004;12(5 Suppl 1):S248-60.

2. El-Serag H, Rudolph L. Hepatocellular carcinoma: epidemiology and molecular carcinogenesis. Gastroenterology 2007;132(7):2557-76.
3. Stroffolini T, Andreone P, Andriulli A et al. Characteristics of hepatocellular carcinoma in Italy. J Hepatol 1998;29:944-53.

4. Lee CL, Ko YC. Survival and distribution pattern of childhood liver cancer in Taiwan. Eur $\mathrm{J}$ Cancer 1998;34(13):2064-7. 
5. Crawford JN. Liver and biliary tract. In: Kumar V, Abbas AK, Fausto N, editors. Robbins and Cotran pathologic basis of disease. 7th ed. Philadelphia: Elsevier Saunders; 2005. p. 924-37.

6. Lee JKT, Sagel SS, Stanley RJ, Jay PH. Tomografia computadorizada do corpo com correlação com ressonância magnética. 4a ed. Rio de Janeiro: Guanabara Koogan; 2010. Portuguese.

7. Miyoshi A, Kitahara K, Kohya N, Noshiro H, Miyazahi $\mathrm{K}$. Outcomes of patients with spontaneous rupture of hepatocellular carcinoma. Hepatogastroenterology. 2011;58(105):99-102.

8. Liu CL, Fan ST, Lo CM, Tso WK, Poon RT, Lam CM, Wong
J. Management of spontaneous rupture of hepatocellular carcinoma: single-center experience. J Clin Oncol. 2001;19(17):3725-32.

9. Rossetto A, et al. Treatment of complicated hepatocellular carcinoma. World J Hepatol. 2010;2(1):49-51.

10. Cho SJ,Yoon JH,Hwang SS, Lee HS. Do young hepatocellular carcinoma patients with relatively good liver function have poorer outcomes than elderly patients? J Gastroenterol Hepatol. 2007;22(8):1226-31.

11. Katyal S, Oliver JH 3rd, Peterson MS, Ferris JV, Carr BS, Baron RL. Extrahepatic metastases of hepatocellular carcinoma. Radiology. 2000;216(3):698-703.

\section{Conflito de interesse: Não}

Submetido em: 12 de Maio de 2011

Aceito em: 23 de Maio de 2011

Correspondência: Serviço de Iconologia

Av. Prof. Lineu Prestes, 2565 - Cidade Universitária - São Paulo - SP - Brasil

CEP: 05508-000 - Tel.: +55.11. 3091.9301

E-mail: jasmartines@gmail.com 